\title{
Daily dialysis reduces pulse wave velocity in chronic hemodialysis patients
}

\author{
Lucia Di Micco, Serena Torraca, Maria Luisa Sirico, Domenico Tartaglia and Biagio Di Iorio
}

Pulse wave velocity (PWV) is a predictor of morbidity and mortality in patients with end-stage renal disease (ESRD). Dialysis patients show cyclic changes in PWV related to their hydration status and blood pressure. Our aim is to assess the impact of daily dialysis on PWV. We performed a randomized crossover study of 60 patients who underwent standard hemodialysis (HD) three times per week for at least 6 months. Patients were classified into three groups according to their PWV values before (pre-) and after (post-) HD, with a cutoff value of $12 \mathrm{~m} \mathrm{~s}^{-1}$, as follows: the low-low (LL) group had normal pre-HD and post-HD PWV; the high-low (HL) group had high pre-HD PWV and normal post-HD PWV; and the high-high (HH) group had high pre- and post-HD PWV. All patients continued standard HD for 2 weeks. A total of 10 patients from each group were randomly assigned to continue standard HD for 1 week and then underwent daily dialysis for 1 week. The remaining 10 patients underwent daily dialysis for 1 week and then underwent standard HD for 1 week. PWV values were measured before and $1 \mathrm{~h}$ after each dialysis session. With daily dialysis treatment, 2 of 20 patients (10\%) moved from the PWV-HH group to the PWV-HL group, whereas 18 of 20 patients $(90 \%)$ moved from the PWV-HL group to the PWV-LL group $(P=0.030)$. Daily dialysis reduces PWV in the ESRD patients. As PWV is a strong predictor of mortality in ESRD and has cyclic variations in patients who are on standard HD, we believe that daily dialysis may be used in patients with high PWV levels to reduce their mortality risk. Hypertension Research (2012) 35, 518-522; doi:10.1038/hr.2011.230; published online 26 January 2012

Keywords: daily hemodialysis; morbidity; mortality; pulse wave velocity

\section{INTRODUCTION}

Cardiovascular (CV) disease is the leading cause of mortality in patients with end-stage renal disease (ESRD). The identification of $\mathrm{CV}$ risk factors, the extent to which they are predictive of mortality, and the identification of patients who could benefit from early interventions are of paramount importance in the management of ESRD. Aortic stiffness, which is assessed through aortic pulse wave velocity (PWV), is a predictor of morbidity and mortality in the general population ${ }^{1-4}$ and in ESRD patients. ${ }^{5,6}$ We previously demonstrated that hemodialysis (HD) patients have weekly cyclic variations in PWV that correlate with their dialysis rhythms, hydration status and blood pressure (BP). ${ }^{7}$ In addition, we observed for the first time that different $\mathrm{PWV}$ behaviors during dialysis were associated with different mortality risks. ${ }^{8}$ Patients with high PWV values before and after HD had an increased mortality risk, compared with those with low PWV values before and after HD, and those with high pre-dialysis PWV levels and low post-dialysis levels. ${ }^{8}$ However, several studies have shown that daily dialysis reduces patient mortality compared with intermittent dialysis (three times per week), improving CV instability, fluid overload, BP, electrolyte balance and poor clinical metabolic conditions. ${ }^{9-12}$

Our aim is to assess the impact of daily dialysis on cyclic variations of PWV in HD patients.

\section{METHODS}

We performed a controlled, prospective, crossover, randomized study.

The inclusion criteria were ESRD with a urinary output $<200 \mathrm{ml}$ per day, treatment with chronic standard bicarbonate HD for at least 6 months (three HD sessions of $4 \mathrm{~h}$ per week), and steady dry weight achieved in the previous 3 months (assessed by either inferior vena cava diameter or bioelectrical impedance analysis). The exclusion criteria were acute CV accidents and infections in the previous 3 months, advanced heart failure (New York Heart Association stages III or IV), atrial fibrillation or arrhythmias, pacemaker implantation, liver disease or ascites, acute or neoplastic illness, overt edema, dysautonomic disease and diabetes. Patients were classified into three groups on the basis of their PWV values, as described previously. ${ }^{7}$ The first group included patients with PWV values $<12 \mathrm{~m} \mathrm{~s}^{-1}$ before and after HD (low-low: LL). The second group included patients with PWV values $>12 \mathrm{~m} \mathrm{~s}^{-1}$ before and $<12 \mathrm{~m} \mathrm{~s}^{-1}$ after HD (high-low: HL). The third group included patients with PWV values $>12 \mathrm{~m} \mathrm{~s}^{-1}$ before and after HD (high-high: HH). A cutoff value of $12 \mathrm{~m} \mathrm{~s}^{-1}$ was used, as indicated in a previous study, which provided the first evidence that an increased aortic stiffness (exceeding $12 \mathrm{~m} \mathrm{~s}^{-1}$ ) is a strong independent predictor of an all-cause and mainly of CV mortality in the ESRD patients. ${ }^{13}$

In total, 160 patients were screened. It was possible to match only 60 patients for sex and age into the three study groups. The small number of enrolled patients was sufficient for a correct analysis, because we used a crossover design. We hypothesized that more than $66 \%$ of patients would reduce their PWV values. The study duration was 1 month. The included patients continued the 
4-h HD three times per week for 2 weeks. After this study period, 10 patients from each PWV group were randomly chosen to continue the 4-h HD three times per week for 1 week, and then they underwent daily HD for 1 week (150-180 min, 6 out of 7 days). The remaining 10 patients in the three groups underwent daily dialysis for 1 week, followed by the 4-h HD three times per week for 1 week. The data used for the statistical analysis were collected during the last 2 weeks. Figure 1 shows a diagram of the experimental procedure.

HD sessions were comparable during the study period. For a three-timesper-week dialysis schedule, the dialysis length was $240 \mathrm{~min}$, and $64 \pm 31$ of blood was treated. For a daily dialysis schedule, the dialysis length was $180 \mathrm{~min}$, and $63 \pm 41$ of blood was treated $(P=0.134)$. The dialysis bath composition was similar in both the treatments (sodium: $138-145 \mathrm{mmoll}^{-1}$; calcium: $1.5 \mathrm{mmoll}^{-1}$, magnesium: $0.5 \mathrm{mgl}^{-1}$; potassium: $2-3 \mathrm{mmoll}^{-1}$ ).

The patients underwent dialysis while lying in bed. Measurements were made immediately before (pre) and $1 \mathrm{~h}$ after (post) each dialysis session. BP was measured using a mercury sphygmomanometer; the first and fifth Korotkoff sounds were used to identify systolic BP (SBP) and diastolic BP, respectively. The mean of three consecutive BP measurements was recorded and used for the statistical analysis. Body weight was detected using a bed balance, using a scale with a sensitivity of $0.05 \mathrm{~kg}$ (Tassinari, Cento, Italy). The carotid-femoral PWV was measured with an automated system (Pulse Pen, DiaTecne, Milan, Italy) according to the foot-to-foot method. ${ }^{7}$ Carotid and femoral waveforms were acquired simultaneously with two pressure-sensitive transducers, and the transit time of the pulse was calculated by the system software program. The distance between the two arterial sites was measured on the body, using a tape measure, and the PWV was calculated as the distance divided by time (meters per second). At least 12 successive readings were used for the analysis to cover a complete respiratory cycle. All PWV measurements were performed by the same operator, and the mean of three consecutive measurements (taken 5 min apart) was used for statistical analyses. If the coefficient of variation (Co-V) for PWV was $>5 \%$, the patient was excluded from the study (none of the patients showed Co-V for PWV >5\%). The observed PWV (PWV-o) was measured and normalized (PWV-n) to the SBP and heart rate (HR) according to the following formula: PWV-n=PWV-o $\times($ SBP-n/SBP-o $) \times(H R-n / H R-o)$, where SBP- $n$ is the normalized SBP (reference value $=130 \mathrm{~mm} \mathrm{Hg}$ ), SBP-o is the observed SBP, HR- $n$ is the normalized HR (reference value $=72$ beats per min) and HR-o is the observed HR. The PWV-n was used for the statistical analysis. It is important to consider that when the PWV-o was normalized for BP and $\mathrm{HR}$, the cyclic changes in PWV were unmodified, with only minor changes in single values during each of the repeated measurements. ${ }^{7}$

Pre-dialysis serum creatinine, urea, calcium, ionized calcium, serum phosphates, sodium, potassium, bicarbonates and hemoglobin levels were measured at baseline and at the end of the study. Serum albumin, blood lipids, plasma fibrinogen, C-reactive protein and parathyroid hormone levels were measured

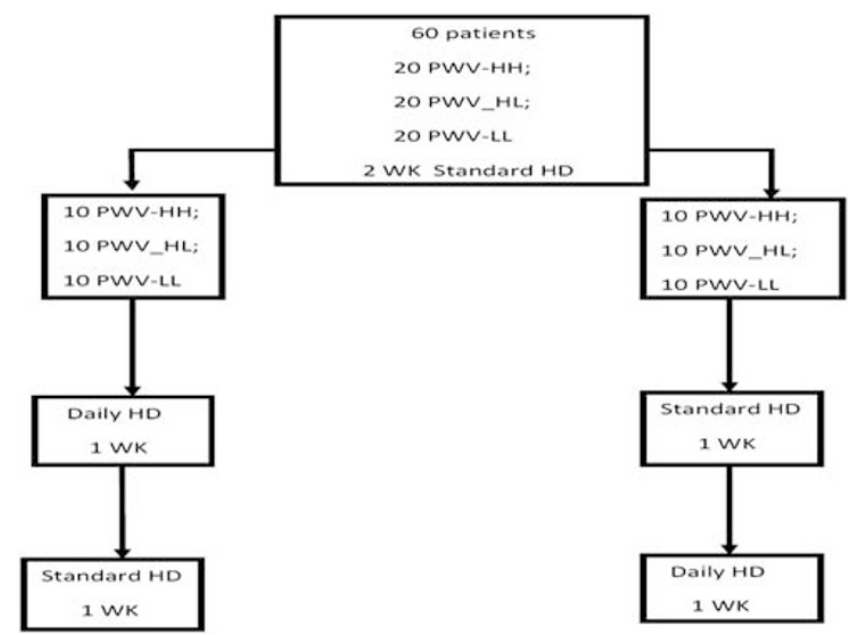

Figure 1 Diagram of the experimental procedure. at baseline. CV history and pharmacological therapy were recorded at baseline for each patient. The coronary artery calcification score was assessed at baseline by multi-slice, light-speed equipment (GE Medical System, Chicago, IL, USA) ${ }^{14}$ Investigators were instructed to achieve the therapeutic targets according to current clinical practice guidelines. The target $\mathrm{BP}$ was $\leqslant 130 / 80 \mathrm{~mm} \mathrm{Hg}$ and the target hemoglobin levels and transferrin saturation were $11 \mathrm{~g} \mathrm{dl}^{-1}$ and $20 \%$ or above, respectively. The target levels for glycated hemoglobin ( $\mathrm{Hb} 1 \mathrm{c}$ ), total cholesterol, low-density lipoprotein cholesterol and triglycerides were less than $7 \%, 200,100$ and $180 \mathrm{mg} \mathrm{dl}^{-1}$, respectively. Sampling was performed during the second session of the week, which is in line with the center's policy to obtain the correct dialysis dosage. The blood sample that was used to determine the final plasma urea concentration was drawn from the arterial line $10 \mathrm{~s}$ after the reduction of the blood flow to $100 \mathrm{ml} \mathrm{min}^{-1}$, with dialysate flow in bypass and by clamping the venous line after the venous bubble trap. The estimated $\mathrm{Kt} / \mathrm{V}$ and equilibrated protein catabolic rate values were calculated using procedures and simplified equations according to Daugirdas. ${ }^{15}$

The ethics committee of ASL AVELLINO approved the study according to the Helsinki Declaration, and all patients gave written informed consent.

\section{Statistics}

All values are reported as the means \pm s.d. Analysis of variance was used to compare the different groups. The differences in frequencies were determined by a $\chi^{2}$-analysis. A multivariate regression analysis was used to analyze the changes in PWV, adjusting for baseline PWV, pre-dialysis BP and ultrafiltration rates. Variable significance was defined as $P<0.05$, which was adjusted for all variables in the final model.

\section{RESULTS}

Table 1 shows the data of all patients in the study group. The three groups were homogeneous for sex, age, BP and serum chemistry (phosphorus, calcium, hemoglobin, parathyroid hormone, albumin, low-density lipoprotein cholesterol and C-reactive protein). The $\mathrm{HH}$ group had an elevated prevalence of ASCVD (cerebrovascular disease, peripheral vascular disease, history of angina pectoris and history of myocardial infarction; $P<0.001)$. The coronary artery calcification score $(P<0.0001)$ and the number of anti-hypertensive drugs

Table 1 Demographic and clinical characteristic of study patients

\begin{tabular}{|c|c|c|c|c|}
\hline & LLPWV & $H L P W V$ & HH PWV & \\
\hline Number & 20 & 20 & 20 & \\
\hline Males (\%) & 60 & 60 & 60 & NS \\
\hline Age (years) & $63.3 \pm 16.6$ & $66.8 \pm 16.8$ & $63.2 \pm 16.5$ & NS \\
\hline ASCVD (\%) & 30 & 40 & 75 & 0.001 \\
\hline Hypertension (\%) & 75 & 75 & 75 & NS \\
\hline SBP (mm Hg) & $139 \pm 22$ & $141 \pm 28$ & $144 \pm 29$ & NS \\
\hline DBP (mm Hg) & $77 \pm 12$ & $78 \pm 11$ & $80 \pm 12$ & NS \\
\hline Phosphorous (mg dl ${ }^{-1}$ ) & $4.1 \pm 1.5$ & $4.0 \pm 1.4$ & $3.9 \pm 1.5$ & NS \\
\hline Calcium (mg dl-1) & $9.1 \pm 0.7$ & $8.9 \pm 1.0$ & $9.0 \pm 1.1$ & NS \\
\hline Hemoglobin $\left(\mathrm{g} \mathrm{dl}^{-1}\right)$ & $11.3 \pm 1.1$ & $11.2 \pm 1.0$ & $11.1 \pm 1.1$ & NS \\
\hline Intact-PTH (pg mmol-1) & $234 \pm 196$ & $212 \pm 200$ & $253 \pm 297$ & NS \\
\hline Albumin $\left(\mathrm{gdl}^{-1}\right)$ & $3.8 \pm 0.5$ & $4.2 \pm 0.6$ & $4.0 \pm 0.7$ & NS \\
\hline LDL-cholesterol (mg dl-1) & $111 \pm 29$ & $101 \pm 26$ & $93 \pm 48$ & NS \\
\hline $\mathrm{CRP}\left(\mathrm{mg} \mathrm{dl} \mathrm{d}^{-1}\right)$ & $7.1 \pm 9.6$ & $8.5 \pm 11.4$ & $9.1 \pm 13.4$ & NS \\
\hline CAC & $286 \pm 544$ & $381 \pm 773$ & $832 \pm 1221$ * & 0.0001 \\
\hline $\mathrm{PWV}\left(\mathrm{ms}^{-1}\right)$ & $7.6 \pm 1.9 * *$ & $15.1 \pm 2.1$ & $17.8 \pm 2.0$ & 0.001 \\
\hline Anti-hypertensive drugs & $2.3 \pm 1.8$ & $2.7 \pm 1.7$ & $3.9 \pm 2.0 *$ & 0.001 \\
\hline
\end{tabular}

Abbreviations: ASCVD, cerebrovascular disease, peripheral vascular disease, history of angina pectoris, history of myocardial infarction; CAC, coronary artery calcium score; CRP, C-reactive protein; DBP, diastolic blood pressure; HH, high-high; HL, high-low; LDL, low-density lipoprotein; LL, low-low; NS, not significant; PTH, parathyroid hormone; PWV, pulse wave velocity before dialysis; SBP, systolic blood pressure; UF, ultrafiltration rate. ${ }^{*} P<0.05 \mathrm{HH}$ vs. LL and $\mathrm{HL}$. ${ }^{*} P<0.05 \mathrm{HH}$ vs. $\mathrm{LL}$ and $\mathrm{HL}$.
${ }^{*} P<0.05 \mathrm{LL}$ vs. $\mathrm{HL}$ and $\mathrm{HH}$. 
Table 2 Dialytic parameters during standard (240-min 3-weekly dialysis) and daily dialysis (150 or 180 -min 6-weekly dialysis)

\begin{tabular}{|c|c|c|c|}
\hline & Standard dialysis & Daily dialysis & P-values \\
\hline Dry weight & $70 \pm 4$ & $66 \pm 5$ & 0.0001 \\
\hline $\mathrm{Qb}\left(\mathrm{ml} \mathrm{min}^{-1}\right)$ & $266 \pm 27$ & $348 \pm 38$ & 0.0001 \\
\hline Blood, (I) & $64 \pm 3$ & $63 \pm 4$ & 0.121 \\
\hline $\mathrm{Na}$ after dialysis $\left(\mathrm{mmol} \mathrm{I}^{-1}\right)$ & $137 \pm 6$ & $139 \pm 4$ & 0.034 \\
\hline UF $(\mathrm{ml} \mathrm{min}-1)$ & $10.5 \pm 1.6$ & $5.1 \pm 1.2$ & 0.0001 \\
\hline SBP before dialysis $(\mathrm{mm} \mathrm{Hg})$ & $137 \pm 19$ & $119 \pm 24$ & 0.0001 \\
\hline SBP after dialysis $(\mathrm{mm} \mathrm{Hg})$ & $121 \pm 33$ & $122 \pm 24$ & 0.85 \\
\hline DBP before dialysis $(\mathrm{mm} \mathrm{Hg})$ & $80 \pm 12$ & $72 \pm 11$ & 0.0001 \\
\hline DBP after dialysis $(\mathrm{mm} \mathrm{Hg})$ & $77 \pm 19$ & $74 \pm 24$ & 0.449 \\
\hline Weekly Kt/V & $4.2 \pm 1.3$ & $4.6 \pm 1.2$ & 0.082 \\
\hline
\end{tabular}

Abbreviations: DBP, diastolic blood pressure; SBP, systolic blood pressure; UF, ultrafiltration rate. Bold values indicate significant $P$ values.

$(P<0.001)$ were significantly higher in the HH group. The antihypertensive therapy that was used before the beginning of the study was continued for the entire month; angiotensin-converting-enzyme inhibitors were used in $70 \%$, sartanics in $60 \%$, beta-blockers in $50 \%$, calcium-antagonist in $25 \%$, and others in $25 \%$ of the patients. In total, $20 \%$ of the patients received two anti-hypertensive drugs, $40 \%$ received three drugs, $30 \%$ received four drugs, and $10 \%$ received five drugs.

Table 2 shows the difference between dialysis three times per week and daily dialysis in all patients: dry weight, ultrafiltration rate, predialysis SBP and pre-dialysis diastolic BP were significantly reduced during daily dialysis compared with dialysis administered three times per week.

At baseline, in the PWV-HH group, four patients had PWV between 12 and $14 \mathrm{~m} \mathrm{~s}^{-1}$, nine patients had PWV between 14 and $18 \mathrm{~m} \mathrm{~s}^{-1}$, and seven patients had PWV $>18 \mathrm{~m} \mathrm{~s}^{-1}$. In the PWV-HL group, three patients had PWV between 12 and $14 \mathrm{~ms}^{-1}$, eight patients had PWV between 14 and $18 \mathrm{~m} \mathrm{~s}^{-1}$, and nine patients had $\mathrm{PWV}>18 \mathrm{~m} \mathrm{~s}^{-1}$. Figure 1a shows the pre- and post-dialysis PWV measurements of the study participants obtained during the threetimes-per-week dialysis treatment. The PWV-HH group had an average PWV of $14.75 \pm 2.2 \mathrm{~m} \mathrm{~s}^{-1}$; the PWV-HL groups had an average value of $11.23 \pm 3.4 \mathrm{~m} \mathrm{~s}^{-1}$; and the PWV-LL group had an average PWV of $9.23 \pm 1.7 \mathrm{~m} \mathrm{~s}^{-1} \quad(P<0.0001$; post-hoc Bonferroni analysis $\mathrm{PWV}-\mathrm{HH} P<0.05$ vs. other groups; PWV-HL before HD vs. after $\mathrm{HD}, P<0.05)$. Figure 2 shows the pre- and post-dialysis $\mathrm{PWV}$ measurements of subjects during their daily dialysis treatments as follows: the PWV-HH group had an average PWV of $14.68 \pm 2.4 \mathrm{~ms}^{-1}$; the PWV-HL group had an average PWV of $9.21 \pm 2.1 \mathrm{~m} \mathrm{~s}^{-1}$; and the PWV-LL group had an average PWV of $8.96 \pm 1.9 \mathrm{~m} \mathrm{~s}^{-1}(P<0.0001$; post-hoc Bonferroni analysis PVW-HH $P<0.05$ vs. other groups). During daily dialysis treatment (Figure 3), 2 of 20 patients (10\%) moved from the PWV-HH group to the PWV-HL group (one patient had a baseline PWV value between 12 and $14 \mathrm{~m} \mathrm{~s}^{-1}$, and one had a baseline PWV value between 14 and $18 \mathrm{~m} \mathrm{~s}^{-1}$ ), whereas 18 of 20 patients (90\%) moved from the PWV-HL group to the PWV-LL group (three patients had baseline PWV between 12 and $14 \mathrm{~ms}^{-1}$, eight patients had baseline PWV between 14 and $18 \mathrm{~m} \mathrm{~s}^{-1}$, and seven patients had baseline PWV $\left.>18 \mathrm{~m} \mathrm{~s}^{-1} ; P=0.030\right)$. Patients in the PWV-LL group showed low levels of PWV during the daily dialysis.

Table 3 shows the predictors associated with the variations in PWV values by the means of multiple logistic regression, and the PWV

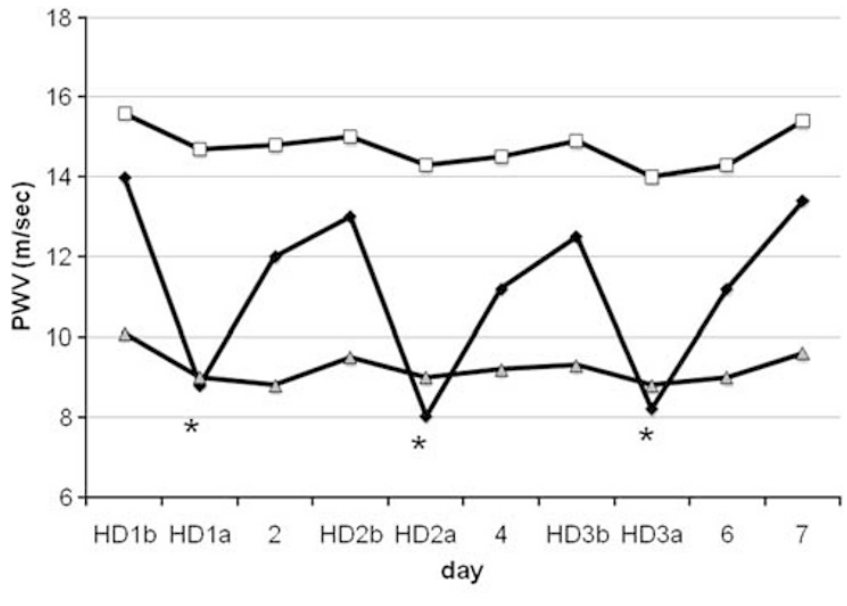

$\rightarrow \mathrm{PWV}-\mathrm{HL} \leadsto \mathrm{PWV}-\mathrm{HH} \rightarrow \mathrm{PWV}-\mathrm{LL}$

Figure 2 Pulse wave velocity (PWV) in subjects during dialysis three times per week. Hemodialysis (HD) $1=$ first weekly hemodialysis, HD2=second weekly hemodialysis, HD3=third weekly hemodialysis, $B=P W V$ measurement before dialysis, $A=P W V$ measurement after dialysis, ${ }^{*} P<0.001$.

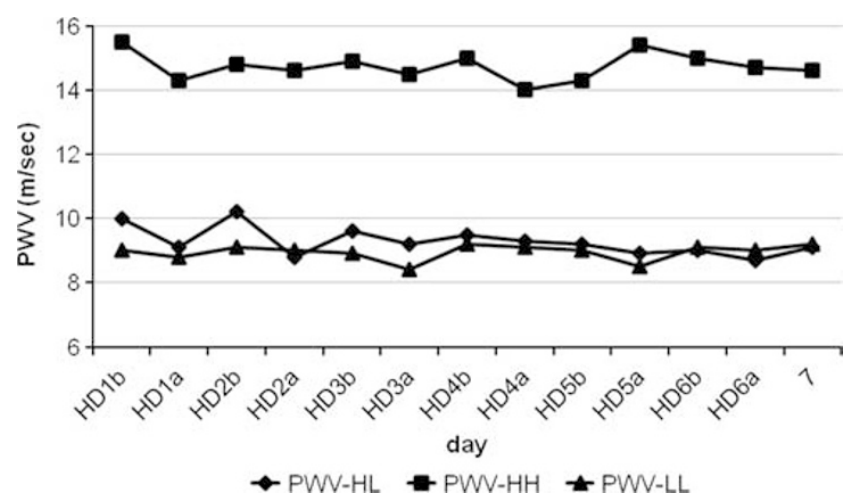

Figure 3 Pulse wave velocity (PWV) in subjects during daily dialysis. Hemodialysis (HD) $1=$ first weekly hemodialysis, HD2=second weekly hemodialysis, HD3=third weekly hemodialysis, HD4=fourth weekly hemodialysis, HD5=fifth weekly hemodialysis, HD6=sixth weekly hemodialysis, $B=P W V$ measurement before dialysis, $A=P W V$ measurement after dialysis.

Table 3 Predictors associated to change of PWV values by means of multiple logistic regression

\begin{tabular}{lccc}
\hline & B coefficient & OR $(95 \%$ Cl) & P-values \\
\hline PWV baseline $\left(\mathrm{m} \mathrm{s}^{-1}\right)$ & -0.39 & $0.94(0.88-0.99)$ & 0.03 \\
Pre-dialysis SBP $(\mathrm{mm} \mathrm{Hg})$ & 0.11 & $1.11(0.77-1.26)$ & $\mathrm{NS}$ \\
Pre-dialysis DBP (mm Hg) & 0.09 & $099(0.87-1.21)$ & $\mathrm{NS}$ \\
UF rates (I per HD) & 0.10 & $1.04(0.91-1.31)$ & $\mathrm{NS}$ \\
Daily HD (yes vs. no) & -0.47 & $0.78(0.69-0.85)$ & 0.01 \\
\hline
\end{tabular}

Abbreviations: $\mathrm{Cl}$, confidence interval; DBP, diastolic blood pressure; HD, hemodialysis; PWV, pulse wave velocity; OR, odds ratio; SBP, systolic blood pressure; UF, ultrafiltration rate.

variations depend from low baseline PWV values $(P=0.03)$ and daily dialysis $(P<0.01)$, pre-SBP. Pre-SBP values and the ultrafiltration rate were not significant. 


\section{DISCUSSION}

Our group showed for the first time that individual PWV levels in dialysis patients undergo cyclic changes during each weekly period. PWV decreased during the dialysis sessions and increased during the interdialytic periods, ${ }^{7}$ and three different $\mathrm{PWV}$ behaviors were defined; some patients had high levels of PWV before dialysis (using a cut-off value of $12 \mathrm{~m} \mathrm{~s}^{-1}$ ) that remained high after dialysis; some patients had high pre-dialysis PWV values that became normal after dialysis; and other patients had normal levels of PWV both before and after dialysis. ${ }^{7}$ We also showed that different PWV behaviors due to the cyclic changes that were observed during dialysis are related to a difference in mortality in dialysis patients, with higher mortality in the patients with increased PWV before and after HD. ${ }^{8}$ Moreover, there was a significant decrease in aortic PWV when a three-times-per-week dialysis schedule was replaced with daily dialysis. In fact, $10 \%$ of PWV-HH patients moved to the PWV-HL group, whereas $90 \%$ of PWV-HL patients reduced their PWV to below $12 \mathrm{~m} \mathrm{~s}^{-1}$ after undergoing daily dialysis. Notably, all patients were treated with both dialysis schedules to obtain the same values of blood volume treated $(P=0.121)$ and dialytic efficiency (weekly Kt/V; $P=0.082)$. Pre-dialysis SBP and dry weight were lower $(P<0.0001)$ with daily dialysis. We observed a reduction in the aortic PWV after 1 week of daily dialysis treatment. Our hypothesis is that, before the arterial wall alterations become structural, characterized by a loss of elastin fibers and an increase in collagen, there is a functional phase during which a treatment aimed to reduce the PWV could allow the recovery of the arterial wall. Calcifications are probably an indirect sign of the transition from functional damage to structural changes of the arterial wall; ${ }^{16}$ in fact, patients from the PWV-HH group had a significantly higher prevalence of ASCVD and higher coronary artery calcification score compared with the other two groups. A better control of fluid overload, which is obtained with slower ultrafiltration during daily dialysis, contributes to the normalization of PWV. A reduction of $4 \mathrm{~kg}$ in the dry weight was obtained in the same patient after switching from standard HD to daily HD. ${ }^{17-19}$ However, fluid overload control is not the only explanation for a reduction in dry weight. Agarwal and Light, ${ }^{17}$ who studied 11833 BP measurements from 125 HD patients, showed that the median weight gain was $2.4 \mathrm{~kg}$ over an interdialytic period, which would result in a $5-\mathrm{mm} \mathrm{Hg}$ increase in systolic pressure, a 2-mm Hg increase in diastolic pressure, and a 3-mm $\mathrm{Hg}$ increase in pulse pressure. This change would be in addition to an increase of $6.2 \mathrm{~mm} \mathrm{Hg}$ in systolic pressure, $3.5 \mathrm{~mm} \mathrm{Hg}$ in diastolic pressure, and $3.1 \mathrm{~mm} \mathrm{Hg}$ in pulse pressure, if no weight gain occurred. Thus, interdialytic weight gain has a substantial role in the interdialytic increases in BP, but there is a great variability in the influence of weight gain on BP. Therefore, the role of daily dialysis on the reninangiotensin-aldosterone system should also be considered. ${ }^{19}$

Our results are of great importance, because we studied high-risk patients who could benefit from a specific intervention and who might be studied in future randomized, controlled trials. In fact, the majority of patients receive a 4-h HD three times per week, according to a calculation of dialysis adequacy. With HD three times per week, the relatively long interval between dialysis sessions results in a 'peak-andvalley' effect, which is characterized by fluctuations in the levels of toxins and body fluid volume. ${ }^{20}$ Several studies have shown that increasing the frequency of dialysis improves outcomes such as anemia, left ventricular hypertrophy, calcium and phosphorus balance and health-related quality of life. Therefore, shorter but more frequent dialysis sessions appear to be a potential solution to this problem. ${ }^{21,22}$

On the contrary, several reports have suggested that cyclic changes in body weight and BP, which are typically observed when undergoing
HD three times per week, influence arterial stiffness and PWV values. ${ }^{17,18,23}$ Prospective epidemiological studies have drawn attention to the arterial elastic properties as independent and strong CV risk factors and predictors of all-cause and CV death. ${ }^{13,24-29}$ The role of the arterial system is to dampen the pressure oscillations and obtain a stable flow to the peripheral tissues. The efficiency of this function depends on the elastic properties of the arterial walls and the geometries of the arteries. In normal conditions, approximately $10 \%$ of the energy produced during systole is stored in the arterial walls. During diastole, the energy stored recoils the elastic arteries, squeezing the stored blood forward into the peripheral tissue. When the arterial system is rigid, this mechanism does not function, and the stroke volume flows through the peripheral tissues only during systole. ${ }^{30}$

Our study has some limitations, including the following: (1) a small sample size, (2) a short duration of follow-up, and (3) a lack of information about smoking status. Despite the small number of patients included in this study, we used a crossover design to increase the power of the study. The follow-up duration was short, because we aimed to study the early changes in PWV due to daily dialysis. Further studies are needed to confirm our data and to assess the long-term effects of daily dialysis on PWV.

In conclusion, we showed for the first time that daily dialysis reduces PWV values. As we used low-cost and easily available tools to identify and treat high-risk patients, this method should be considered in daily clinical practice to predict adverse outcomes and reduce mortality in this subgroup of ESRD patients.

\section{CONFLICT OF INTEREST}

The authors declare no conflict of interest.

1 Benetos A, Safar M, Rudnichi A, Smulyan H, Richard JL, Ducimetieère P, Guize L. Pulse pressure: a predictor of long-term cardiovascular mortality in a French male population. Hypertension 1997; 30: 1410-1415.

2 Hayashi T, Nakayama Y, Tsumura K, Yoshimaru K, Ueda H. Reflection in the arterial system and the risk of coronary heart disease. Am J Hypertens 2002; 15: 405-409.

3 Weber T, Auer J, O'Rourke MF, Kvas E, Lassnig E, Berent R, Eber B. Arterial stiffness, wave reflections, and the risk of coronary artery disease. Circulation 2004; 109: 184-189.

4 Hansen TW, Staessen JA, Torp-Pedersen C, Rasmussen S, Li Y, Dolan E, Thijs L, Wang JG, O'Brien E, Ibsen H, Jeppesen J. Ambulatory arterial stiffness index predicts stroke in a general population. J Hypertens 2006; 24: 2247-2253.

5 Blacher J, Asmar R, Djane S, London GM, Safar ME. Aortic pulse wave velocity as a marker of cardiovascular risk in hypertensive patients. Hypertension 1999; 33. 1111-1117

6 Blacher J, Guerin AP, Pannier B, Marchais SJ, Safar ME, London GM. Impact of aortic stiffness on survival in end-stage renal disease. Circulation 1999; 99: 2434-2439.

7 Di lorio B, Nazzaro P, Cucciniello E, Bellizzi V. Influence of haemodialysis on variability of pulse wave velocity in chronic haemodialysis patients. Nephrol Dial Transplant 2010; 25: 1579-1583.

8 Torraca S, Sirico ML, Guastaferro P, Morrone LF, Nigro F, Blasio AD, Romano P, Russo D, Bellasi A, Di lorio B. Variability of pulse wave velocity and mortality in chronic haemodialysis patients. Hemodial Int 2011; 15: 326-333.

9 Buoncristiani U. Fifteen years of clinical experience with daily haemodialysis. Nephrol Dial Transplant 1998; 13(S6): 148-151.

10 Kjellstrand CM, Buoncristiani U, Ting G, Traeger J, Piccoli GB, Sibai-Galland R, Young BA, Blagg CR. Short daily haemodialysis: survival in 415 patients treated for 1006 patients-years. Nephrol Dial Transplant 2008; 23: 3283-3289.

11 Kliger AS. More intensive haemodialysis. Clin J Am Soc Nephrol 2009; 4(Suppl 1): s121-s124.

12 Twardowski ZJ, Misra M. 'Daily' dialysis: lesson from a randomized, controlled trial. $N$ Engl J Med 2010; 363: 2363-2364.

13 Raggi P, Bellasi A, Ferramosca E, Islam T, Muntner P, Block GA. Association of pulse wave velocity with vascular and valvular calcification in haemodialysis patients. Kidney Int 2007; 71: 802-807.

14 Di lorio B, Nargi O, Cucciniello Bellizzi V, Torraca S, Russo D, Bellasi A, INDEPENDENT study investigators. Coronary artery calcification progression is associated with arterial stiffness and cardiac repolarization deterioration in haemodialysis patients. Kidney Blood Press Res 2011; 34: 180-187. 
15 Daugirdas JT. Simplified equations for monitoring Kt/V, PCRn, eKt/V and ePCRn. Adv Ren Replace Ther 1995; 2: 295-304.

16 Safar ME, Blacher J, Pannier B, Guerin AP, Marchais SJ, Guyonvarc'h PM, London GM. Central pulse pressure and mortality in ESRD. Hypertension 2002; 39: 735-738.

17 Agarwal R, Light RP. Arterial stiffness and interdialytic weight gain influence ambulatory blood pressure patterns in haemodialysis patients. Am J Physiol-Renal Physio 2008; 294: F303-F308.

18 Vuurmans JL, Boer WH, Bos WJ, Blankestijn PJ, Koomans HA. Contribution of volume overload and angiotensin II to the increased pulse wave velocity of haemodialysis patients. J Am Soc Nephrol 2002; 13: 177-183.

19 Hopkins K, Bakris GL. Assessing blood pressure control in dialysis patients: finally a step forward. Hypertension 2009; 53: 448-449.

20 Kjellstrand CM, Evans RL, Petersen RJ, Shideman JR, Von Hartitzsch B, Buselmeier TJ. The 'unphysiology' of dialysis: a major cause of dialysis side effects? Hemodial Int 2004; 8: 24-29.

21 Perl J, Chan CT. Home haemodialysis, daily haemodialysis, and nocturnal hemodialysis: core curriculum 2009. Am J Kidney Dis 2009; 54: 1171-1184.

22 Kaysen GA, Larive B, Painter P, Craig A, Lindsay RM, Rocco MV, Daugirdas JT, Schulman G, Chertow GM, FHN Trial Group. Baseline physical performance, health, and functioning of participants in the frequent haemodialysis network trail. Am J Kidney Dis 2011; 57: 101-112.
23 Safar ME, Asmar R, Benetos A, Levy BI, London GM. Sodium, large arteries, and diuretic compounds in hypertension. Am J Med Sci 1994; 307(Suppl 1): s3-s8.

24 Gusbeth-Tatomir P, Covic A. Causes and consequences of increased arterial stiffness in CKD patients. Kidney Blood Press Res 2007; 30: 97-107.

25 Munakata M, Sakuraba J, Tayama J, Furuta T, Yusa A, Nunokawa T, Yoshinaga K, Toyota T. Higher brachial-ankle pulse wave velocity is associated with more advanced carotid atherosclerosis in ESRD. Hypertens Res 2005; 28: 9-14.

26 Blacher J, Guerin AP, Pannier B, Marchais SJ, London GM. Arterial calcifications, arterial stiffness, and cardiovascular risk in ESRD. Hypertension 2001; 38: 938-942.

27 Blacher J, Safar ME, Guerin AP, Pannier B, Marchais SJ, London GM. Aortic pulse wave velocity index and mortality in end-stage renal disease. Kidney Int 2003; 63: 1852-1860.

28 Heerspink HJ, Ninomiya T, Zoungas S, de Zeeuw D, Grobbee DE, Jardine MJ, Gallagher M, Roberts MA, Cass A, Neal B, Perkovic V. Effect of lowering blood pressure on cardiovascular events and mortality in patients on dialysis: a systematic review and meta-analysis of randomised trials. Lancet 2009; 373: 1009-1015.

29 Inrig JK, Patel UD, Toto RD, Reddan DN, Himmelfarb J, Lindsay RM, Stivelman J, Winchester JF, Szczech LA. Decreased pulse pressure during haemodialysis is associated with improved 6-month outcomes. Kidney Int 2009; 76: 1098-1107.

30 London GM, Pannier B. Arterial functions: how to interpret the complex physiology. Nephrol Dial Transplant 2010; 25: 3815-3823. 